\title{
Evaluation of HAAH/humbug quantitative detection in the diagnosis of hepatocellular carcinoma
}

\author{
TIAN XUE ${ }^{1}$, JING SU ${ }^{2}$, HONGMIN LI ${ }^{1}$ and XIAOPING XUE ${ }^{3}$ \\ ${ }^{1}$ Faculty of Life Science, Northwest University, Xi'an, Shaanxi 710069; ${ }^{2}$ Department of Microbiological Test, \\ Nantong Center for Disease Control and Prevention, Nantong, Jiangsu 226007; ${ }^{3}$ Department of Microbiology, \\ The Fourth Military Medical University, Xi'an, Shaanxi 710072, P.R. China
}

Received July 25, 2014; Accepted October 3, 2014

DOI: $10.3892 /$ or.2014.3606

\begin{abstract}
Human aspartyl-(asparaginyl)- $\beta$-hydroxylase(HAAH) is a type 2 transmembrane protein and an $\alpha$-ketoglutaratedependent dioxygenase that can stereospecifically catalyze the post-translational hydroxylation reaction of $\beta$-carbon atoms of aspartic acid and asparagine residues present in epidermal growth factor-like domains of certain specific proteins. Humbug is a truncated isoform of aspartyl (asparaginyl) $\beta$-hydroxylase that lacks the catalytic domain. A series of reports demonstrated that overexpression of $\mathrm{HAAH} /$ humbug was identified in hepatocellular carcinoma (HCC) and various tumor tissues. However, the prognostic value of HAAH/humbug expression in HCC remains unclear. The purpose of this study was to investigate the expression of the HAAH/humbug gene at the mRNA and protein levels in $\mathrm{HCC}$ and to assess the overexpression of HAAH/humbug as a diagnostic and prognostic marker in HCC. HAAH/humbug mRNA levels were measured in 120 HCCs and 40 paired nontumor liver tissues by molecular beacon (MB) quantitative RT-PCR. Immunohistochemical staining was used to detect the protein level of the HAAH/humbug in the same specimens. ROC analysis was performed based on the expression levels of the HAAH/humbug gene in 120 cases of HCC tissues and 40 cases of adjacent non-tumor liver tissues. The results showed that 117 (97.5\%) of the 120 frozen sections of patients with HCCs had HAAH/humbug-positive immunoreactivity, whereas the 40 adjacent non-tumor liver tissues exhibited no staining. Higher levels of HAAH/humbug mRNA were found
\end{abstract}

Correspondence to: Dr Hongmin Li, Faculty of Life Science, Northwest University, 229 North Taibai Road, Xi'an, Shaanxi 710069, P.R. China

E-mail: lihm2006@126.com

Professor Xiaoping Xue, Department of Microbiology, The Fourth Military Medical University, 17 Changlexi Street, Xi'an, Shaanxi 710032, P.R. China

E-mail: xxp890825@sina.com

Key words: human aspartyl-(asparaginyl)- $\beta$-hydroxylase, hepatocellular carcinoma, molecular beacon, quantitative RT-PCR in $114(95 \%)$ of the $120 \mathrm{HCC}$ tissues relative to the adjacent cancer-free tissue. ROC curve analysis exhibited that the sensitivity was $90.1 \%$, specificity was $97.6 \%$ and ROC AUC was 0.986 . The specific value of $\mathrm{HAAH} / \beta$-actin abundance used as a cut-off point was 0.315 , while the gene copy number (7.35 copies $/ \mu 1$ ) was used a as cut-off point, with sensitivity being $99.2 \%$, specificity $96.7 \%$ and the ROC AUC used 0.990 . No statistically significant difference was observed for these two factors. HAAH/humbug expression levels were upregulated in almost all the HCC tissues when compared to the adjacent cancer-free tissue, irrespective of the cut-off point used. Results of the present study suggested that HAAH/ humbug is a potential diagnostic and prognostic biomarker for the treatment of HCC.

\section{Introduction}

Human aspartyl-(asparaginyl)- $\beta$-hydroxylase (HAAH) is a type 2 transmembrane protein and an $\alpha$-ketoglutaratedependent dioxygenase that can stereospecifically catalyze the post-translational hydroxylation reaction of $\beta$-carbon atoms of aspartic acid and asparagine residues present in epidermal growth factor-like domains of certain specific proteins (1). The $H A A H$ gene was first reported by Korioth et al (2). It is positioned on q12 of chromosome No. 8, and the full length of mRNA is $2449 \mathrm{bp}$. The alternative splicing of the $H A A H$ gene generates at least five homeotic subfamily mRNA transcripts $\mathrm{a}, \mathrm{b}, \mathrm{c}, \mathrm{d}$ and e, which encode the proteins $A A H$, Junctate, Humbug, Junctin and Junctin-1, respectively (3-5). AAH is a unique protein with enzymatic activity, and Junctate, Junctin and Junctin-1, which are mainly expressed on the endoplasmic reticulum membrane and the sarcoplasm membrane of the myocardium diaphragm, are calcium-binding proteins connected with the instantaneous release of calcium ions, and with protein modification in the process of protein synthesis. Humbug is a truncated isoform of HAAH that lacks the catalytic domain. Humbug also participates in calcium regulation and increases intracellular calcium levels by promoting calcium release from intracellular stores. In recent years, using immunohistochemistry, gene expression analysis, in vivo binding and enzyme-linked immunosorbent assay, it has been confirmed that HAAH/humbug is overexpressed in a broad range of malignant tumor tissues and cell lines and it 
is not expressed or has a low expression in normal, non-cancer inflammatory lesion and paracancerous tissues. Over 1,000 cases of malignant tumor specimens including cholangiocarcinoma; hepatocellular carcinoma (HCC); prostate, breast, colon, lung, gastric cancer and ovarian cancer; pancreatic carcinoma; glioblastoma; oligodendroglioma and primitive neuroectodermal tumors were previously detected, exhibiting a positive rate of $>95 \%$ or even $>99 \%$ (6-10). Previous study results showed that humbug has been associated with a variety of human cancers, and although humbug lacks enzymatic activity, it is expressed at levels comparable with that of HAAH in various cancer cell lines. Humbug was also useful in the evaluation of HCC prediction and prognosis $(5,11)$. In addition, as a transmembrane protein, HAAH/humbug can be dissociated from the surface of cancer cells and is readily shed into the blood of cancer patients. Free HAAH in the blood serum of 857 cases of lung, breast, prostatic and colon cancer and non-tumor serum samples, was quantitatively determined. The sensitivity of the test was $94.7 \%(n=857)$, specificity was $94.3 \%(n=211)$ and overall accuracy was $94.6 \%$. The average values of free HAAH in the blood serum were $0 \mathrm{ng} / \mathrm{ml}$ (noncancer), $25.7 \mathrm{ng} / \mathrm{ml}$ (prostate cancer), $34.6 \mathrm{ng} / \mathrm{ml}$ (lung cancer), $17.6 \mathrm{ng} / \mathrm{ml}$ (breast cancer) and $30.0 \mathrm{ng} / \mathrm{ml}$ (colon cancer) (12). The present study showed that HAAH is a special carcinoembryonic antigen and an important marker in the formation of malignant cells. HAAH is overexpressed in a variety of malignant tumor tissues and transformed cell lines. As a new tumor marker, HAAH has a broad spectrum and specificity (13). In the preliminary study, we conducted a qualitative analysis on the distribution and expression of HAAH in seven types of tumor cell lines and 20 tumor tissues including liver cancer, cholangiocarcinoma, kidney and breast cancer. The present study indicates that HAAH mRNA was overexpressed in all seven (100\%) tumor cell lines examined, although the levels of the gene expression varied and the positive rate was $90.4 \%$ (94/104) in 20 tumor tissues (14).

RT-PCR (mRNA detection) and northern blotting (protein detection) are frequently used to detect the expression level of HAAH mRNA and protein. However, these methods have certain drawbacks, such as a long operation time, are prone to false-positive or -negative results, deliver low diagnostic yields and are only used for qualitative detection. The molecular beacon (MB) method, however, is simple, rapid and highly specific, and has been used for the quantitative detection of various types of tumor-marker genes.

In the present study, we examined the expression levels of the HAAH/humbug gene and proteins in tissue specimens of patients with HCC and adjacent HCC-free liver tissues, to determine whether the HAAH/humbug gene and protein overexpression levels are directly associated with HCC clinicopathologically and to evaluate the overexpression of the $H A A H$ gene and protein as a diagnostic and prognostic biomarker in $\mathrm{HCC}$.

\section{Materials and methods}

Patients and tissue specimens. A total of 120 surgically resected tissue specimens from patients with HCC and 40 adjacent HCC-free liver tissue were obtained from The First Affiliated Hospital of Medical College of Xi'an Jiaotong
University and the Xijing Hospital of Digestive Diseases, Fourth Military Medical University, respectively. None of these patients underwent radiotherapy or chemotherapy prior or subsequent to surgical resection. The study included 84 males and 36 females, with an age range of 35-70 years (median 55.1 \pm 8.2 years). The samples were obtained in accordance with the Ethics requirements for conducting medical experiments. These samples were tested and diagnosed at the Department of Pathology, Xijing Hospital.

Main reagents. TRIzol, reverse transcriptase M-MLV, RNase inhibitor, quantitative PCR kit (SuperMix-UDG) were purchased from Invitrogen Life Technologies, Carlsbad, CA, USA, and anti-HAAH mAb was prepared in our laboratory (15).

RT-PCR primer and HAAH/humbug $M B$. Three pairs of RT-PCR primers, FP1/RP1, FP2/FRP2 and FP/RP, were designed using Oligo 6.0 analysis software. Primer FP1 contained the restriction endonuclease sites XhoI, Kozak sequence and His-tag sequence. Primer RP1 contained stop code TAA and the SpeI restriction endonuclease sites. Products from the primer pair FP1/RP1 were applied to amplify HAAH/humbug regions that were used for the construction of recombinant plasmid carrying the humbug gene fragment. Primer pair FP2/RP2 was utilized for the detection of the HAAH/humbug gene in the MB quantitative RT-PCR (RT-qPCR) reaction. Primer pair FP/RP was used to amplify the housekeeping gene $\beta$-actin. Humbug molecular beacon probes (humbug MB, a sequence of 27 nucleotides, which can recognize a target molecule) and $\beta$-actin MB (a sequence of 30 nucleotides, which can recognize a target molecule) were designed using Beacon Designer 7.0 software. Their target specificity was verified using BioEdit ver. 7.0.9.0 software. The primers and MB probe above were synthesized by Sangon Co. (Shanghai, China) (Table I).

Specimen collection and RNA extraction. Fresh surgically resected HCC tissue samples and paracancerous HCC-free liver tissue specimens were collected. These fresh samples were rapidly transferred and stored in liquid nitrogen prior to RNA purification. Total RNA from snap-frozen tissue was extracted using TRIzol reagent (Invitrogen Life Technologies) according to the manufacturer's instructions. The integrity of the total RNA was validated using UV spectrophotometry and $20 \mathrm{~g} / 1$ agarose gel electrophoresis. Only high-quality RNA with intact $18 \mathrm{~S}$ and $28 \mathrm{~S}$ RNA was used for subsequent experiments, and then stored at $-80^{\circ} \mathrm{C}$ until use.

Preparation of HAAH/humbug cDNA. RNA samples were reverse-transcribed using the M-MLV First Strand cDNA synthesis kit (Roche, Basel, Switzerland). Oligo-dT18 was used for primers of the first chain synthesis. cDNA was reversetranscribed from $2 \mu \mathrm{g}$ of the extracted RNA in a $20 \mu \mathrm{l}$ reaction system containing $2 \mu \mathrm{l}$ of $5 \mathrm{X}$ reaction buffer, $1 \mu \mathrm{l}$ of M-MLV reverse transcriptase, $1 \mu \mathrm{l}$ of RNAsin, $2 \mu \mathrm{l}$ of $0.1 \mathrm{M}$ DTT, $1 \mu \mathrm{l}$ of Oligo-dT 18 primers $(50 \mu \mathrm{g} / \mathrm{ml}), 6 \mu \mathrm{l}$ of dNTP mix $(10 \mathrm{mM})$ and DEPC-treated water. The samples were heated to $70^{\circ} \mathrm{C}$ for $5 \mathrm{~min}$, followed by incubation at $42^{\circ} \mathrm{C}$ for $1 \mathrm{~h}$. Reactions were terminated by heating at $95^{\circ} \mathrm{C}$ for $5 \mathrm{~min}$. $\beta$-actin mRNA levels 
Table I. Sequences of molecular beacon and primers.

\begin{tabular}{llcc}
\hline Name of primer/MB & \multicolumn{1}{c}{ Sequence (5'-3') } & Gene position (bp) & Amplified fragments (bp) \\
\hline Humbug FP1 & CCATCGATGCCACCATGTGGGCCATCAT & $175-195$ & 766 \\
& CATCATCATCATATGGTGATTGCATTGCTGGGC & & \\
Humbug FP2 & GGACTAGTTTATGTTTCTGGTGGTAC & $922-939$ & 267 \\
Humbug FP2 & ATGGTGATTGCATTGCTGGGC & $186-206$ & \\
Humbug RP2 & CAGAATATCGAAGATGAAGCA & $432-452$ & \\
Humbug MB & FAM-AGCAGGTTCCTGTGGAGGCAGAACCC & $413-440$ & 114 \\
$\beta$-actin FP & CAGAATACCTGCT-DABCYL & & \\
$\beta$-actin RP & TGGCATTGCTGACAGGATG & $227-245$ & \\
$\beta$-actin MB & AAGTACTCCGTGTGGATCG & $331-350$ & \\
& FAM-CCGCATTGGCTCCCAGCACCATGAAG & $266-294$ & \\
\hline
\end{tabular}

measured in parallel reactions were used to calculate the relative abundance of each mRNA transcript.

Construction of the recombinant plasmid carrying an HAAH/humbug-encoding gene. cDNA obtained by reverse transcription was used as a template for the polymerase chain reaction (PCR). The primer pair humbug FP1/RP1 was used for the amplification reaction. The total volume of $50 \mu \mathrm{l}$ contained $5 \mu \mathrm{l}$ of cDNA template, $5 \mu 1$ 10X PCR Buffer $\left(\mathrm{Mg}^{2+}\right.$ Plus), $1 \mu 1$ primer humbug FP1 and RP1 each, $4 \mu 110 \mathrm{mM}$ dNTPs, $0.5 \mu 1 \mathrm{rTaq}$ DNA polymerase (Dalian Takara Co.), and $33.5 \mu 1$ ultrapure water. The initial denaturation step at $95^{\circ} \mathrm{C}$ for 5 min was followed by 30 cycles at $94^{\circ} \mathrm{C}$ for $1 \mathrm{~min}$, $60^{\circ} \mathrm{C}$ for $1 \mathrm{~min}, 72^{\circ} \mathrm{C}$ for $1 \mathrm{~min}$, followed by $72^{\circ} \mathrm{C}$ for $10 \mathrm{~min}$ (Mastercycler Gradient, Eppendorf Co., Hamburg, Germany). PCR products were then separated on a $1.2 \%$ agarose gel containing $5 \mu \mathrm{l} / 100 \mathrm{ml}$ Goldview using DL2000 ladder (Shanghai Shinegene Co., Shanghai, China) as a size marker. The PCR products were cloned into pMD18-T vector using a TA cloning kit (Promega Co., Madison, WI, USA) and sequenced. The recombinant plasmid was identified using the restriction enzyme digestion method. The concentration and purity of the recombinant plasmid DNA were estimated by UV. DNA was diluted in $0.9 \% \mathrm{NaCl}$ at a concentration of $1 \mathrm{mg} / \mathrm{ml}$. The ratio of OD260/280 $\mathrm{nm}$ ranged 1.8-2.0. The plasmid DNA was stored at $-20^{\circ} \mathrm{C}$ in $0.5-\mathrm{ml}$ aliquots.

Sensitivity experiment and linear standard curve. According to the manufacturer's instructions, AccuBlue ${ }^{\mathrm{TM}}$ dsDNA quantification kits were used to have a precise quantitative detection on the pMD-Humbug plasmid DNA that had the HAAH/humbug genes (uQuant Microplate Scanning spectrophotometer; Bio-Tek, Winooski, VT, USA). The precise quantitative plasmid DNA was used as the template. Sterile deionized water was used to achieve gradient dilution, and the concentration gradients were $10^{5}, 10^{4}, 10^{3}, 10^{2}, 10^{1}$ and $10^{\circ}$ (copies $/ \mu 1)$. The control was pMD empty plasmid diluted with sterile deionized water. A $25 \mu \mathrm{l}$ reaction system was utilized with $2.5 \mu 1$ of $10 \mathrm{X}$ PCR buffer, $0.25 \mu 1$ of dNTP $(10 \mathrm{mmol} / \mathrm{l})$, $6 \mu \mathrm{l}$ of $\mathrm{MgCl}_{2}(25 \mathrm{mmol} / \mathrm{l}), 0.5 \mu \mathrm{l}$ for Primer humbug FP2 and RP2 $(10 \mu \mathrm{mol} / 1)$, respectively, $0.5 \mu \mathrm{l}$ of Taq DNA polymerase (5 U/ $\mu 1$, Dalian Takara Co.), $1 \mu \mathrm{l}$ of pMD-Humbug plasmid template and $0.25 \mu \mathrm{l}$ of Humbug $\mathrm{MB}(10 \mu \mathrm{mol} / \mathrm{l})$. The reaction conditions were $95^{\circ} \mathrm{C}$ for $5 \mathrm{~min}, 95^{\circ} \mathrm{C}$ for $45 \mathrm{sec}, 55^{\circ} \mathrm{C}$ for $60 \mathrm{sec}$, $72^{\circ} \mathrm{C}$ for $60 \mathrm{sec}$ for 45 cycles. Experiments were performed in triplicate and repeated twice. Following the reaction, the fluorescent quantitative PCR analysis software was used to set the baseline and cycle threshold $(\mathrm{Ct})$ and quantitative amplification curves were obtained. The $\mathrm{Ct}$ value was used as the abscissa and ordinate, with the fluorescence intensity value as ordinate, the linear standard curve cycle threshold $(\mathrm{Ct})$ as abscissa, and the logarithms of DNA concentration as the ordinate.

Immunohistochemical staining. Cryostat sections of frozen tissue were cut at $6 \mu \mathrm{m}$ (freezing microtome; Leica CM1950, Nussloch, Germany), placed on glass slides, air dried and fixed in a 1:1 solution of alcohol:acetone. The slides were then washed gently with PBS 2-3 times and were submerged in methanol containing $10 \% \mathrm{H}_{2} \mathrm{O}_{2}$ to quench endogenous peroxidase activities in tissues. Following washing and air drying, the sections were stored at $-20^{\circ} \mathrm{C}$ until use. Immediately before commencement of immunostaining, the sections were washed in PBS buffer for $5 \mathrm{~min}$ and treated with 5\% skimmed milk to block non-specific bindings on the sections. The slides were rinsed with PBS buffer to remove the blocking solution and air dried again. The anti-HAAH MAbs (also recognizing humbug) were then added and incubated in a moist chamber at $37^{\circ} \mathrm{C}$ for $45 \mathrm{~min}$. The slides were rinsed again in PBS buffer. HRP-conjugated anti-mouse antibodies diluted in PBS were added, and incubated in a moist chamber for $45 \mathrm{~min}$ at $37^{\circ} \mathrm{C}$. The slides were washed gently with PBS 3-5 times. TMB blotting substrate was added and developed for $5 \mathrm{~min}$. The sections were gently counterstained with hematoxylin, and then viewed under an inverted microscope. 
$M B$ quantitative RT-PCR. As mentioned above, in the cDNA preparation, total RNA was extracted from tumor tissues, Oligo(dT) 18 was regarded as the reverse transcription primer, and single-stranded cDNA was synthesized under M-MLV reverse transcriptase. The PCR reaction was conducted with this template, and the primer pair FP2 (upstream primer)/ RP2 (downstream primer) and FP (upstream primer)/RT (downstream primer) was used to amplify the HAAH/humbug and $\beta$-actin gene fragments, respectively. The MB RT-qPCR reaction was performed on Chromo $4^{\mathrm{TM}}$ four-color real-time detector (MJ Research Inc., Waltham, MA, USA) to detect the expression levels of the HAAH/humbug genes and housekeeping gene $\beta$-actin in the specimens. The PCR reaction conditions were the same as those for the cDNA preparation. $\beta$-actin RNA levels measured in parallel reactions were used to calculate the relative abundance of each mRNA transcript. After the reaction ended, $5 \mu \mathrm{l}$ of PCR product were used to conduct agarose gel electrophoresis at a concentration of $2.0 \%$. Images were captured on a Gel Doc XR gel-imaging system, and gray was analyzed to the result of electrophoresis.

\section{Results}

Amplification of the HAAH/humbug gene and restriction analysis of the recombinant plasmid pMD-humbug. The complete humbug-encoding gene segment with a molecular weight of $\sim 760$ bp was amplified using RNA extracted from HCC tumor tissue as a template, with FP1/RP1 being used as a RT-qPCR primer (Fig. 1B). However, no DNA fragments of the same size were amplified using RNA isolated from the adjacent tumor-free liver tissues as a template and the same primer pair. The PCR product was gel-purified and then ligated into a pMD18-T vector. The ligation product was incubated overnight at $16^{\circ} \mathrm{C}$ and used for transformation of the E. coli strain DH5 $\alpha$. The transformants were plated on LB agar plates containing $100 \mu \mathrm{g} / \mathrm{ml}$ ampicillin and incubated at $37^{\circ} \mathrm{C}$ for $24 \mathrm{~h}$. Recombinant plasmid was extracted from DH5 $\alpha$ using an EasyExtraction kit (Biofuture Group Inc., Beijing, China). The recombinant plasmid pMD-humbug was identified by $\mathrm{XhoI} / \mathrm{SpeI}$ double restriction enzyme digestion. Restriction digestion analyses clearly showed that there was a specific digestion strip at $\sim 760$ bp in agarose gel electrophoresis and the molecular weight of the digested fragment was consistent with the expected size (Fig. 1A). The positive recombinant plasmid was selected and sequenced (Genscript Co., Nanjing, China). The sequencing result was compared with the HAAH/humbug sequence of the NCBI Genbank database. It was highly homologous to the HAAH/Humbug sequence of the database, except that No. 346 had a G to A mutation, which was the nonsense mutation (data not shown). The positive recombinant plasmid carrying the HAAH/humbug-encoding gene fragments was designated as pMD-humbug.

Sensitivity and specificity of the MB RT-qPCR system. In the $1 \times 10^{0}-1 \times 10^{6} \mathrm{copies} / \mu 1$ range, with an increase in the number of cycles $(\mathrm{Ct})$, the fluorescence intensity of each sample increased at varying degrees. The amplification curve was $\mathrm{S}$-shaped. The values of $\mathrm{Ct}$ and the fluorescence intensity were positively correlated with the initial concentrations of the plasmid DNA
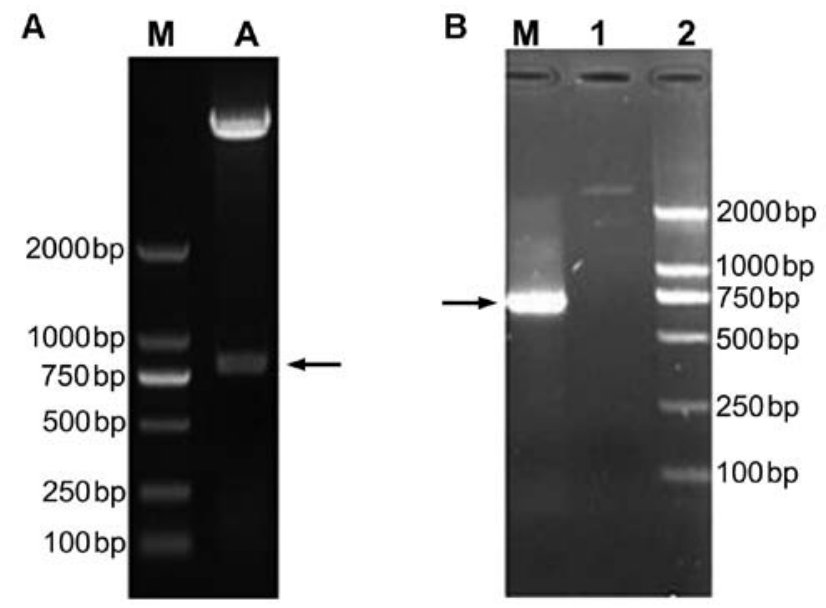

Figure 1. Agarose gel electrophoretic analysis of quantitative RT-PCR (RT-qPCR) products and restriction endonuclease profiles of plasmid of pMD-humbug. (A) Restriction endonuclease profiles of plasmid of pMDhumbug digested with XhoI/SpeI. M, DNA marker DL2000; A, fragment of restriction endonuclease. (B) (RT-qPCR) products of the HAAH/humbug gene. M, DNA marker DL2000; lane 1, cDNA from tumor tissue as template; lane 2, cDNA from adjacent non-tumor tissue as template.

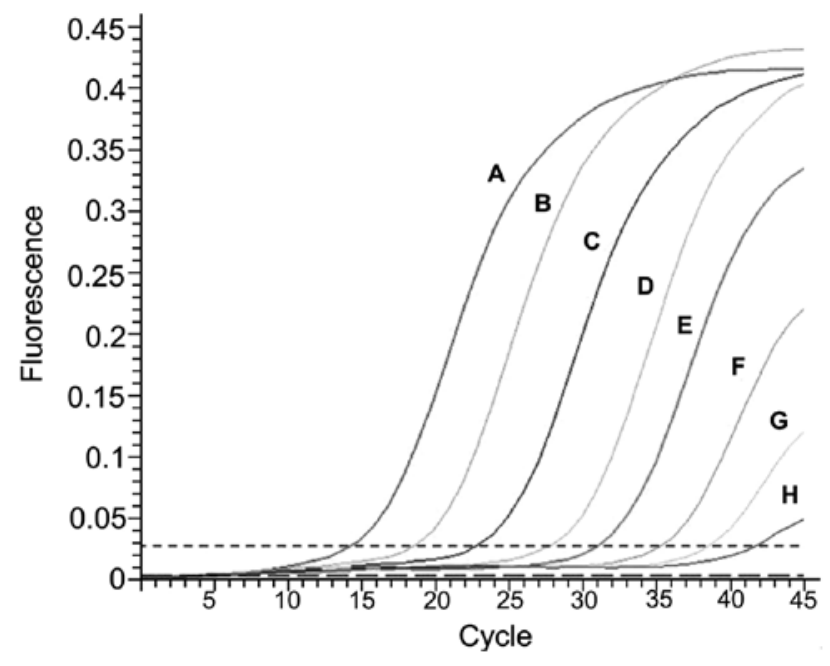

Figure 2. The quantitative amplification kinetic curve of MB quantitative RT-PCR shows known concentrations of recombinant plasmid pMD-humbug DNA as a template. Concentration of plasmid DNA (copies $/ \mu 1$ ) A, 10 $0^{6}$ B , 10 ; $\mathrm{C}, 10^{4}$; D, $10^{3} ; \mathrm{E}, 10^{2} ; \mathrm{F}, 10^{1} ; \mathrm{G}, 10^{0} ; \mathrm{H}$, negative control $\left(10^{3} \mathrm{pMD}-18\right)$.

templates. In the $25-\mu 1$ reaction system, the limit of detection was 1 copy/reaction system (Fig. 2 and Table II).

$M B$ qPCR standard curve of pMD-humbug plasmid. The canonical plotting of MB quantitative RT-PCR of plasmid pMD-humbug is shown in Fig. 3. The logarithm of the standard copy number was regarded as the x-coordinate, and the $\mathrm{Ct}$ value that passed through when fluorescent signal intensity reached the preset threshold was the y-coordinate. A straight standard curve was obtained. In the range of $1 \times 10^{1}-1 \times 10^{6}$ copies $/ \mu 1$, alterations in the number of $\mathrm{Ct}$ indicated a good linear relationship with the concentration of the initial plasmid pMD-humbug DNA, and the calibration curve equation obtained was $\mathrm{Y}=-4.03 \times \log (\mathrm{C})+39.34, \mathrm{r}=0.996$. The DNA samples of unknown concentration were amplified, the 
Table II. Ct value of MB real-time qRT-PCR standard curve.

\begin{tabular}{lccccccc}
\hline $\log (\mathrm{C})$ & $10^{6}$ & $10^{5}$ & $10^{4}$ & $10^{3}$ & $10^{2}$ & $10^{1}$ & $10^{0}$ \\
\hline Run 1 & 14.0 & 20.1 & 21.4 & 26.9 & 29.8 & 33.9 & 41 \\
Run 2 & 14.6 & 17.8 & 25.1 & 28 & 30.7 & 37 & 39.3 \\
Run 3 & 16.4 & 19.1 & 22.5 & 29.1 & 32.5 & 34.1 & 36.7 \\
Mean \pm SD & $15 \pm 1.3$ & $19 \pm 1.2$ & $23 \pm 1.9$ & $28 \pm 1.1$ & $31 \pm 1.3$ & $35 \pm 1.7$ & $39 \pm 2.2$ \\
\hline
\end{tabular}

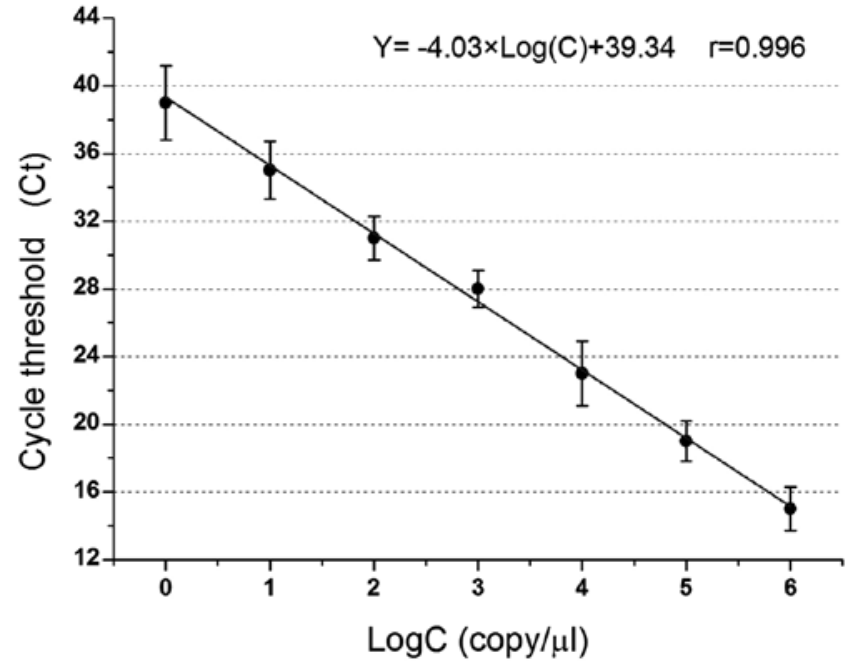

Figure 3. Quantitative standard curve of MB quantitative RT-PCR for pMD-humbug plasmid. Calibration chart for the amplification curves in Fig. 2. Concentration of plasmid DNA (copies $/ \mu 1$ ) $1,10^{6} ; 2,10^{5} ; 3,10^{4} ; 4,10^{3}$; $5,10^{2} ; 6,10^{1}$ and $7,10^{\circ}$.

$\mathrm{Ct}$ values were entered into the calibration curve equation, and the logarithm of the copy number of DNA samples was calculated. The copy number of DNA samples was subsequently obtained through this standard curve.

Analysis of melting curve. Melting curve (Tm) analysis allows for different PCR amplification products and identification of primer dimers according to the difference of melting points in order to differentiate non-specific amplification. Therefore, the influence of non-specific products can be decreased. The ideal melting curve should be a single-peak curve. Two or more peaks indicates that non-specific amplification products, such as primer dimers, are produced in the reaction system. Therefore, Tm analysis was constantly employed to determine PCR primers, PCR products and the specificity of an amplification system. Fig. 4 shows the Tm peak of MB RT-qPCR products of HCC carcinoma tissues highly coincides with that of the products of recombinant plasmid pMD-humbug MB RT-qPCR (positive control), which indicates that the two peaks are identical, and that the MB and primer we designed are of a high specificity.

Expression of the HAAH/humbug gene in HCC tissues. Paired samples of HCC tissues and adjacent HCC-free liver tissues were used to measure HAAH/humbug mRNA levels by RT-qPCR. The $\beta$-actin mRNA levels measured in the

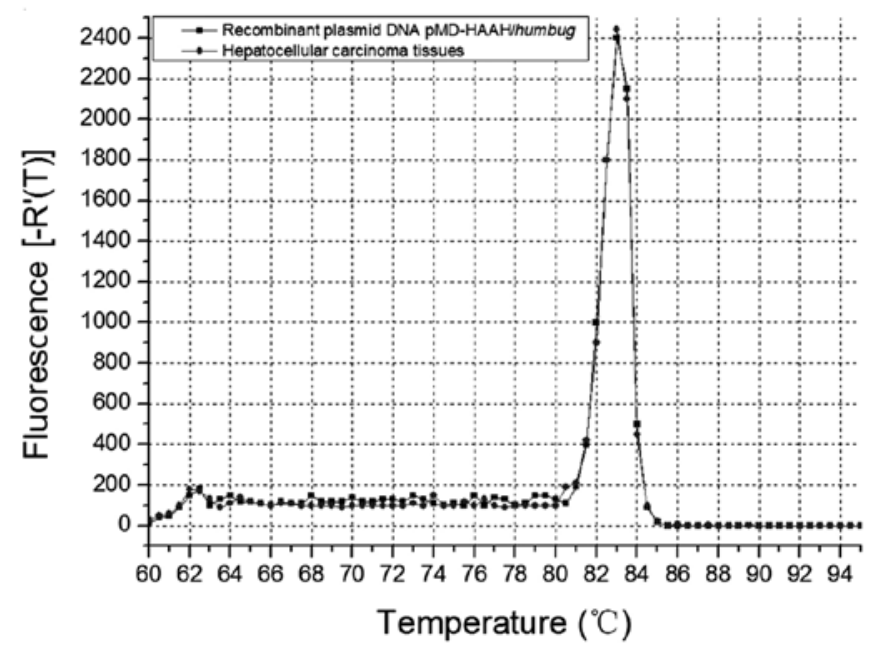

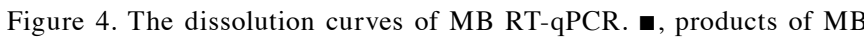
RT-qPCR of recombinant plasmid DNA pMD-HAAH/humbug; $\bullet$, products of qPCR of HCC tissues.

same samples were used to calculate relative HAAH/humbug mRNA abundance (Fig. 5). The intra-group gray value of agarose gel electrophoresis comparisons were performed using Quantity One software (Gel Doc EQ System; Bio-Rad Co., Hercules, CA, USA). MB qPCR studies demonstrated that the expression levels of the HAAH/humbug gene in the HCC tissues and adjacent non-tumor liver tissues were $0.789 \pm 0.287$ and $0.121 \pm 0.098$. The mean level of humbug mRNA was $\sim 5$ - to 8 -fold higher in the cancer samples, compared to the adjacent cancer-free tissues, respectively (Fig. 6).

Immunohistochemical staining analysis of HAAH/humbug in HCC tissues. The results of immunohistochemical (IHC) staining showed that 117 (97.5\%) of the 120 frozen sections of patients with HCCs exhibited HAAH/humbug-positive immunoreactivity, whereas the 40 adjacent non-tumor liver tissues exhibited no staining. The staining pattern for $\mathrm{HAAH} /$ humbug in the tumor cells was primarily cytoplasmic with a distinct perinuclear accentuation and plasmalemmal (Fig. 7).

MB qPCR detection of HAAH/humbug. The MB RT-qPCR studies demonstrated higher levels of HAAH/humbug mRNA in $113(94.2 \%)$ of the 120 cases of the HCC tissues relative to the adjacent cancer-free tissue. The ratio of $\mathrm{HAAH} / \beta$-actin abundance was used as the cut-off point (0.315). Comparatively, only 1 of 40 cases were weakly positive in the adjacent non-tumor liver tissues specimens and the false-positive rate 


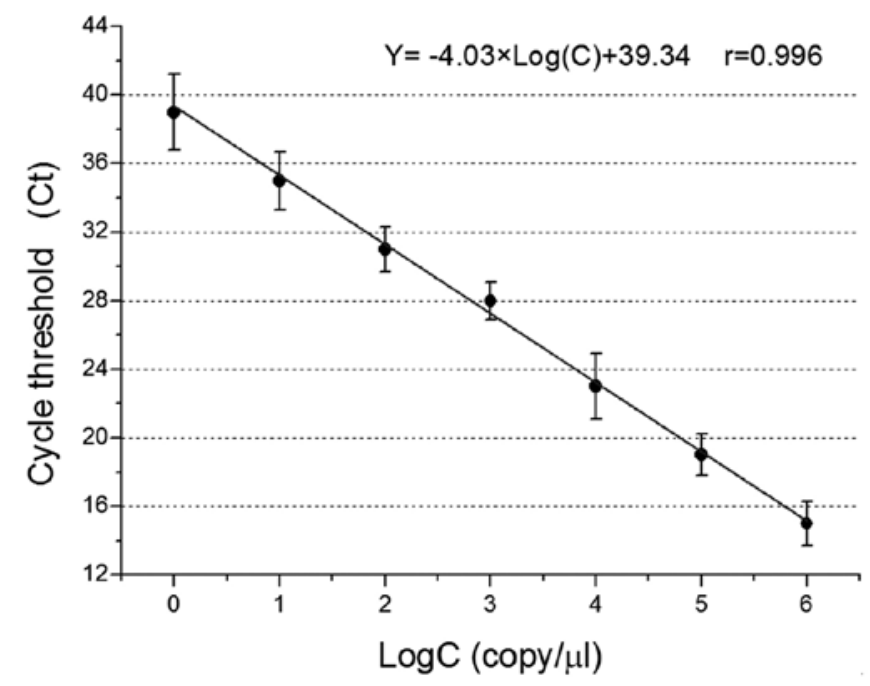

Figure 5. Agarose gel electrophoretic analysis of qPCR products of HAAH/ humbug and internal reference gene $\beta$-actin.

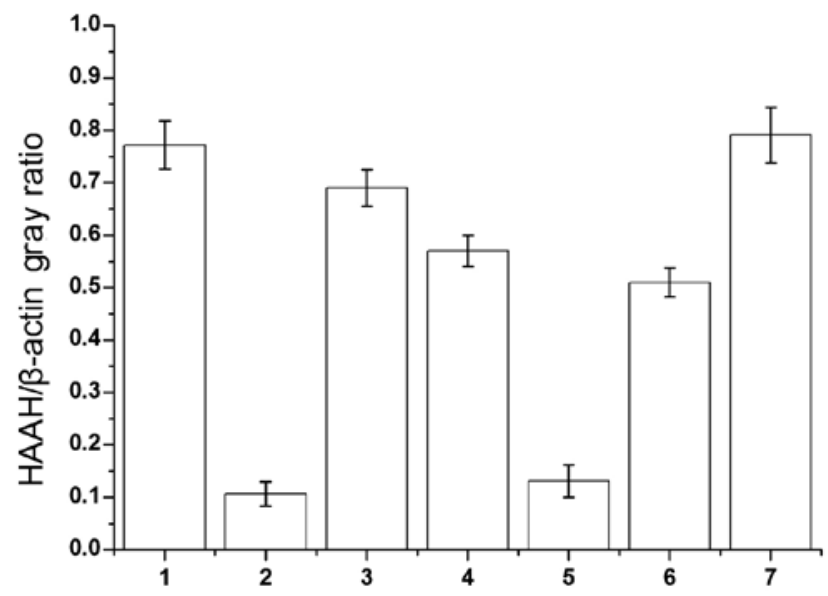

Figure 6. MB qPCR analysis for the expression of HAAH/humbug in HCC tissures and adjacent non-tumor liver tissues. Statistically significant differences were identified between HCC tissues and the corresponding adjacent non-tumor liver tissues $(\mathrm{P}<0.01)$. Bars, mean $\pm \mathrm{SD}$; columns $1,3,4,6$ and 7 , HCC tissues; columns 2 and 5 , adjacent non-tumor liver tissues.
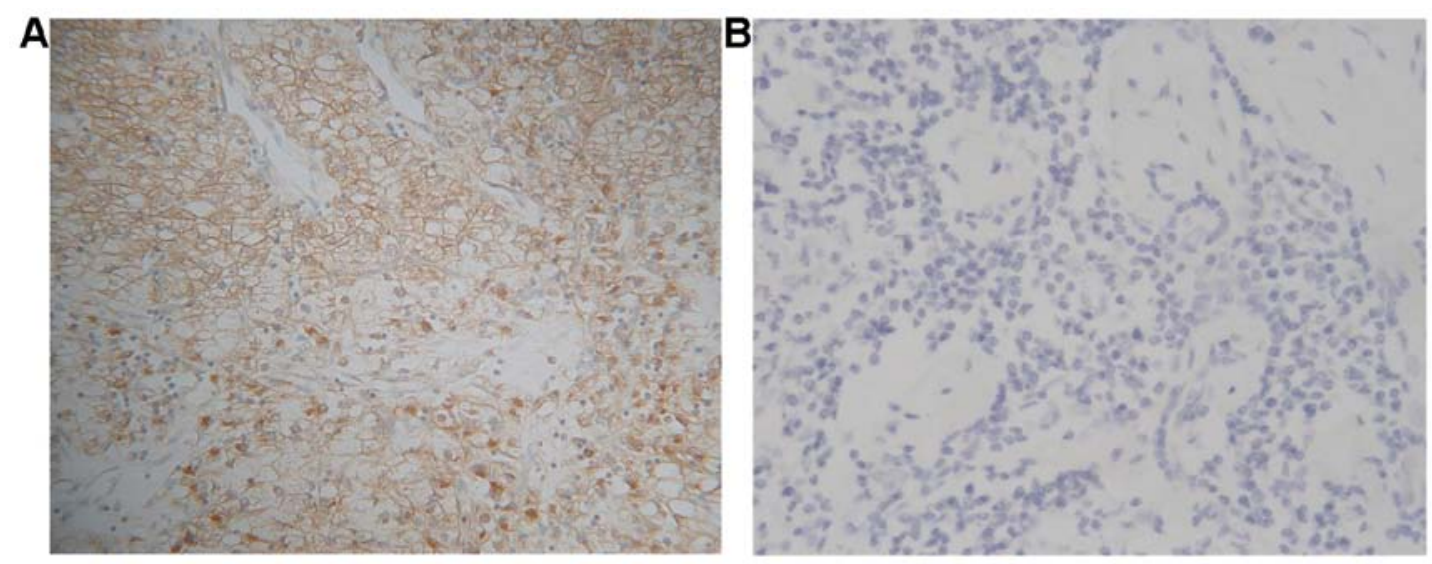

Figure 7. Immunohistochemical staining of HAAH. (A) In the frozen section of HCC tissues and (B) in the frozen section of adjacent HCC-free liver tissues from different patients using against HAAH monoclonal antibody G3/F11 (recognizing humbug). Immunoreactivity was identified by the goat-anti human horseradish peroxidase conjugate using diaminobenzidine (brown precipitate) as the chromogen. Sections were lightly counterstained with hematoxylin to show unlabeled areas. High immunoreactivity was detected on the majority of HCC cells, and localized to the cell surface, cytoplasm, or perinuclear regions (left micrographs; original magnification, x20). Non-tumor hepatic parenchyma was negative or weakly focal-positive (right micrographs; original magnification, x20).

was $2.5 \%$. While the copy number of the HAAH/humbug gene was used as the cut-off point $(77.35 \mu \mathrm{g})$, the positive rate was $114(95 \%)$ of 120 in HCC tissues, and the false-positive rate was also $5 \%$ in the adjacent non-tumor liver tissues. Briefly, the HAAH/humbug expression level was upregulated in almost all the HCC tissue cells relative to normal liver cells, irrespective of the cut-off point used ( $\mathrm{P}<0.01$; Fig. 8).

ROC curve analysis. ROC analysis was performed based on the expression levels of the HAAH/humbug gene in 120 cases of HCC tissues and 40 cases of adjacent non-tumor liver tissues using SPSS 19.0 software (IBM SPSS Statistics Co., Armonk, NY, USA). Fig. 9A shows the result of ROC curve analysis of the ratio of $\mathrm{HAAH} / \beta$-actin abundance. Sensitivity was $90.1 \%$, specificity $97.6 \%$ and ROC AUC was 0.986 with 0.315 copies $/ \mu 1$ as the cut-off point. Fig. 9B shows the gene copy number identified by ROC curve analysis. Sensitivity was

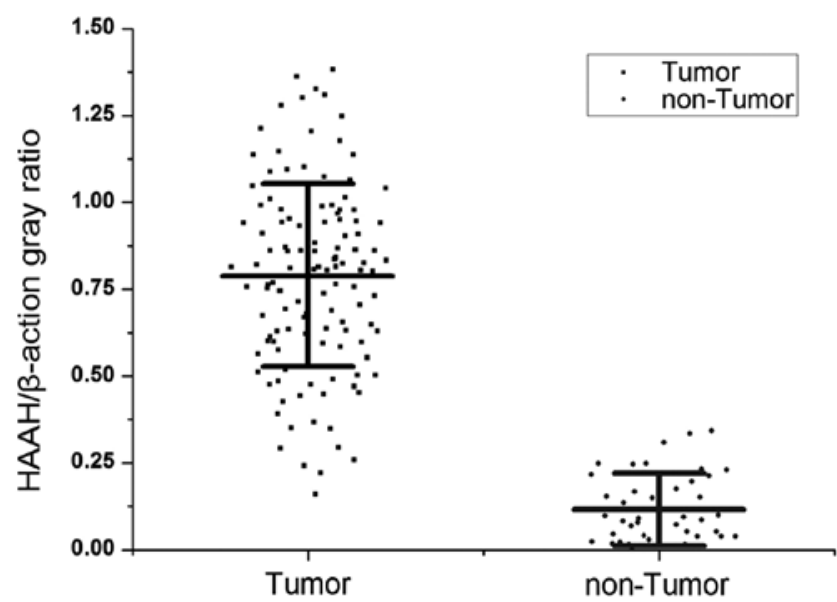

Figure 8. Expression of the $H A A H$ gene in $\mathrm{HCC}$ tissues and adjacent non-tumor liver tissues Graphs are the mean \pm SD values $(0.789 \pm 0.287$, $0.121 \pm 0.098$ ). 

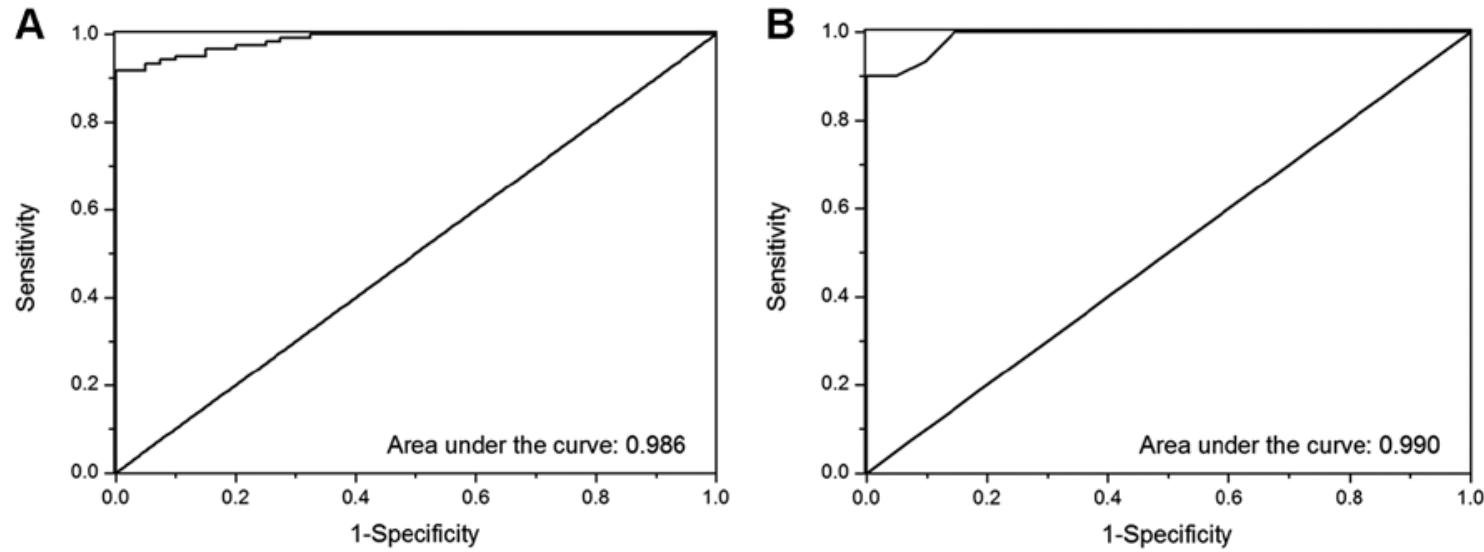

Figure 9. ROC curve analysis for evaluating the diagnostic value of HAAH. (A) ROC curve analysis of the ratio of HAAH/ $\beta$-actin abundance. (B) ROC curve analysis to identify the gene copy number (copies/ $\mu 1)$.

99.2\%, specificity $96.7 \%$ and ROC AUC was 0.990 when the cut-off point was 77.35 copies $/ \mu 1$. No statistically significant difference was noted for the two factors. The results showed that the overexpression of HAAH/humbug has a potential diagnostic and prognostic value for $\mathrm{HCC}$.

\section{Discussion}

HCC is a highly invasive neoplasm with multicentric occurrence, and is also one of the five most prevalent lethal human malignant cancer-types. At present, the mortality rate for HCC ranks third worldwide. In recent years, the HCC incidence rate has been on the increase. The incidence of HCC in North America and Europe is $<10$ in 10 million individual, while its incidence in Asia and Africa is 50-150 in 10 million individuals. China is one of the regions most seriously affected by HCC (16). The annual incidence of $17.83 \%$ accounts for $>40 \%$ of the annual total number of patients with $\mathrm{HCC}$ worldwide. HCC leads to a high mortality rate and is the third leading cause of cancer-related death in China (24,25). It has become the second largest cause of cancer-related mortality in rural areas. Primary HCC is characterised by high invasiveness, insidious onset, no obvious symptoms at the early stage and poor prognosis. Therefore, the early diagnosis, prevention and timely treatment of HCC are particularly important. Numerous approaches, such as liver biopsy puncture, ultrasonography, helical computed tomography, or magnetic resonance imaging, may be applied to detect HCC. As the 'golden standard', $\alpha$-fetoprotein (AFP) is the most common tumor marker in the clinical diagnosis of HCC, but its low sensitivity (70\%), even lower specificity (64\%) when utilized alone in HCC diagnosis easily lead to misdiagnosis and missed diagnosis (17). Therefore, identification of new biomarkers is of great significance for the early diagnosis and early prevention of HCC.

A series of reports $(9,26)$ have documented that the expression levels of HAAH/humbug in HCC tissues is significantly higher than adjacent non-cancerous liver tissues, irrespective of the mRNA or protein levels.

The overexpression of HAAH/humbug increases motility, invasiveness, malignant transformation and the infiltrative proliferation of HCC cells. It has been confirmed that antisense constructs produced against the AUG start codon inhibited gene expression and tumor cell motility and migration (18) and correspondingly, HAAH siRNA exhibited significantly lower mean directional motility indices relative to the control cells. Previous studies have shown that the overexpression of HAAH in transfected normal cells was sufficient to induce cell transformation and suppression of HAAH expression (siRNA) $(9,19)$ or neutralized activity $(\mathrm{mAb})$ that returns cancer cells to a normal phenotype $(20,21)$. The present results indicate that the overexpression of HAAH is closely associated with poor prognosis, tumor recurrence and patient survival (22).

The $H A A H$ gene encodes the proteins AAH, Junctate, Humbug, Junctin and Junctin-1. The N-terminus of HAAH-related proteins has a role in calcium homeostasis, whereas the C-terminal region, which is essential for catalytic activity, is only present in HAAH. As one of the HAAH protein post-translational splicing isoforms, humbug is a truncated homolog of HAAH. It is locked in the domain with the catalytic activity of the enzyme in the C-terminal fragment of the HAAH protein. The molecular weight of the humbug gene is $2.9 \mathrm{~kb}$, encoding $50-55 \mathrm{kDa}$ humbug protein. Although the humbug protein lacks enzymatic activity, it is expressed at levels comparable with that of HAAH in a variety of human cancer types (18)

To assess the value of HAAH/humbug in the diagnosis and prediction of HCC, we examined the expressed levels of the HAAH/humbug gene using MB RT-qPCR and the expressed levels of HAAH protein using immunohistochemical staining in 120 cases of HCC patients and 40 cases of adjacent HCC-free liver tissues.

In the present study, HAAH/humbug expression levels were detected by immunohistochemistry (protein level) and MB RT-qPCR (mRNA level) in 120 tumor tissues of patients with HCC specimens and 40 adjacent non-tumor tissues. The results of immunohistochemistry showed that, in 120 cases of HCC tissue samples, 117 (97.5\%) exhibited positive immunoreactivity for or against HAAH/humbug monoclonal antibodies (mAb), whereas in all 40 cases of adjacent non-cancerous tissues, immunoreactivity was negative. In the experiment of MB RT-qPCR, of $120 \mathrm{HCCs}$, the HAAH/humbug gene expression was strongly positive in 113 tumor tissues (94.2\%), whereas HAAH/humbug mRNA expression was weakly positive in 2 of 40 adjacent non-tumor 
liver tissues (5\%), using the specific value of $\mathrm{HAAH} / \beta$-actin abundance as the cut-off point (0.315). When the copy number of the HAAH/humbug gene $(77.35 \mu \mathrm{g})$ was used as cut-off point, the positive rates were 114 (95\%) of 120 in HCC tissues and upregulated in the majority of HCC tissues relative to the adjacent cancer-free tissue, irrespective of the cut-off point used $(\mathrm{P}>0.05)$.

The following conclusions can be drawn from the aforementioned tests: the HAAH/humbug at the mRNA and protein levels in Chinese HCC patients was markedly higher than that of non-cancerous liver tissues. This finding is consistent with the results of a previous study (20).

ROC analysis was performed based on the expression levels of the HAAH/humbug gene in 120 cases of HCC tissues and 40 cases of adjacent non-tumor liver tissues using SPSS 19.0 software. The results of the ROC curve analysis concerning the ratio of HAAH/ $\beta$-actin abundance showed that the sensitivity was $90.1 \%$, specificity $97.6 \%$ and ROC AUC 0.986 when the cut-off point was 0.315 (Fig. 9A). The results of the ROC curve analysis concerning gene copy number (copies/ $\mu \mathrm{l}$ ) demonstrated that the sensitivity was $99.2 \%$, specificity $96.7 \%$ and ROC AUC was 0.990 when the cut-off point was 77.35 copies/ $\mu$ l (Fig. 9B). No significant difference was identified between these factors. The present study results confirm that HAAH/humbug is extremely valuable for the diagnosis of HCC. Thus, HAAH/humbug serves as a potential diagnostic biomarker for HCC.

In order to improve the sensitivity and specificity of HAAH/humbug mRNA detection on tumor cells, qPCR and hot-start PCR were utilized in previous experiments, although the results obtained were unsatisfactory (data not shown). In this study, the method of MB RT-qPCR was applied to quantitatively measure HAAH/humbug expression levels.

The MB probe technique is a new nucleic acid quantitative detection technique developed by Tyagi and Kramer (23), based on fluorescence energy transfer technology and the linear probe technique. MBs are hairpin-shaped molecules, consisting of a stem loop structure with single-stranded DNA molecules. The MB stem is formed by the attachment, to both termini of the loop, of two short (5-7 nucleotide residues) oligonucleotides that are complementary to each other. The 18-30 bp region of the MB loop in the middle is complementary to the target DNA or RNA and they do not base pair with one another. At the 5 '-end of the stem, a fluorescent dye is covalently attached. The quencher dye is covalently attached to the 3'-end of the stem. When the MB is in closed loop shape, the quencher resides in proximity to the fluorophore, which results in quenching the fluorescent emission of the latter (23). When the MB is bound to a target nucleic, the acid sequence causes the separation of the stem and thus of fluorescence quenching.

The stem of the classical MB comprises complementary sequences unrelated to target sequences. In this study, base pairs at each terminus of the MB stem are complementary to each other, and to the target DNA. After the MB was complemented with the target genes, the space interval between fluorescent dye and the quencher was increased. This was useful for fluorescent emission and to intensify the luminous intensity, therefore, it improves the sensibility of detection. Since there are specific PCR primers and MB probes in this reaction system, the false positivity of detection can be effectively reduced. In this reaction system, the lowest detectable limit (LOD) of HAAH/humbug mRNA was 1 copy/reaction in a $25-\mu 1$ reaction system and the linear detection range was $10^{1}-10^{6}$ copies/reaction.

The strengths of this approach are that it is unnecessary to dilute or concentrate specimens in the experiment, and second that the procedures of detection were completed in a closed tube. The reaction was completed in a closed tube and real-time monitoring of the amplification occurs. Therefore, further handling after the PCR reaction, and the drawback of easy pollution in the conventional PCR was avoided. After MB RT-qPCR was terminated, agarose gel electrophoresis to inspect the products of PCR amplification was not undertaken. When the Ct value of the unknown sample is achieved, the initial sample copy number can be calculated from the standard curve.

Based on the findings of this study, the new MB RT-qPCR method with advantages of high specificity, speed and simplicity can be widely applied for HAAH/humbug mRNA detection in different tumor tissue samples, including early-stage tissue samples.

\section{Acknowledgements}

The authors are grateful for the support offered by the Research Project of Shaanxi Provincial Key Laboratory of Biotechnology (11JS085 and 14JS088) and the Development Project of Science and Technology Research of Shaanxi Province (2011K12-61).

\section{References}

1. Lavaissiere L, Jia S, Nishiyama M, et al: Overexpression of human aspartyl(asparaginyl)beta-hydroxylase in hepatocellular carcinoma and cholangiocarcinoma. J Clin Invest 98: 1313-1323, 1996.

2. Korioth F, Gieffers C and Frey J: Cloning and characterization of the human gene encoding aspartyl beta-hydroxylase. Gene 150: 395-399, 1994.

3. Jones LR, Zhang L, Sanborn K, Jorgensen AO and Kelley J: Purification, primary structure, and immunological characterization of the 26-kDa calsequestrin binding protein (junctin) from cardiac junctional sarcoplasmic reticulum. J Biol Chem 270: 30787-30796, 1995.

4. Treves S, Feriotto G, Moccagatta L, Gambari R and Zorzato F: Molecular cloning, expression, functional characterization, chromosomal localization, and gene structure of junctate, a novel integral calcium binding protein of sarco(endo)plasmic reticulum membrane. J Biol Chem 275: 39555-39568, 2000.

5. Lee JH: Overexpression of humbug promotes malignant progression in human gastric cancer cells. Oncol Rep 19: 795-800, 2008.

6. Dinchuk JE, Henderson NL, Burn TC, et al: Aspartyl beta -hydroxylase (Asph) and an evolutionarily conserved isoform of Asph missing the catalytic domain share exons with junctin. J Biol Chem 275: 39543-39554, 2000.

7. Ince N, de la Monte SM and Wands JR: Overexpression of human aspartyl (asparaginyl) beta-hydroxylase is associated with malignant transformation. Cancer Res 60: 1261-1266, 2000.

8. Maeda T, Taguchi K, Aishima S, et al: Clinicopathological correlates of aspartyl (asparaginyl) beta-hydroxylase overexpression in cholangiocarcinoma. Cancer Detect Prev 28: 313-318, 2004.

9. De la Monte SM, Tamaki S, Cantarini MC, et al: Aspartyl(asparaginyl)-beta-hydroxylase regulates hepatocellular carcinoma invasiveness. J Hepatol 44: 971-983, 2006.

10. Luu M, Sabo E, de la Monte SM, et al: Prognostic value of aspartyl (asparaginyl)-beta-hydroxylase/humbug expression in non-small cell lung carcinoma. Hum Pathol 40: 639-644, 2009.

11. Wang J, de la Monte SM, Sabo E, et al: Prognostic value of humbug gene overexpression in stage II colon cancer. Hum Pathol 38: 17-25, 2007. 
12. Moshiri M, Lebowitz MS and Roberts SF: Cancer biomarker, haah (human aspartyl (asparaginyl) beta-hydroxylase), a companion diagnostic strategy. In: Proceedings of the Fifth Oncology Biomarker Conference. Zurich, p233, 2012.

13. Silbermann E, Moskal P, Bowling N, Tong M and de la Monte SM: Role of aspartyl-(asparaginyl)-beta-hydroxylase mediated notch signaling in cerebellar development and function. Behav Brain Funct 6: 68, 2010.

14. Yang H, Song K, Xue T, et al: The distribution and expression profiles of human Aspartyl/Asparaginyl $\beta$-hydroxylase in tumor cell lines and human tissues. Oncol Rep 24: 1257-1264, 2010.

15. Xue T, Xue XP, Huang QS, Wei L and Sun K: Monoclonal antibodies against human aspartyl (asparaginyl) beta-hydroxylase developed by DNA immunization. Hybridoma 28: 251-257, 2009.

16. Xian ZH, Zhang SH, Cong WM, Yan HX, Wang K and Wu MC: Expression of aspartyl beta-hydroxylase and its clinicopathological significance in hepatocellular carcinoma. Mod Pathol 19: 280-286, 2006

17. Lander ES, Linton LM, Birren B, et al: Initial sequencing and analysis of the human genome. Nature 409: 860-921, 2001

18. Sepe PS, Lahousse SA, Gemelli B, et al: Role of the aspartylasparaginyl-beta-hydroxylase gene in neuroblastoma cell motility. Lab Invest 82: 881-891, 2002.

19. Davila JA, Morgan RO, Shaib Y, McGlynn KA and El Serag HB: Hepatitis Cinfection and the increasing incidence of hepatocellular carcinoma: a population-based study. Gastroenterology 127 1372-1380, 2004.
20. Ho SP, Scully MS, Krauthauser CM, et al: Antisense oligonucleotides selectively regulate aspartyl beta-hydroxylase and its truncated protein isoform in vitro but distribute poorly into A549 tumors in vivo. J Pharmacol Exp Ther 302: 795-803, 2002.

21. Fuller S, Stewart S, Lebowitz M, et al: Immunogenicity of a lambda phage-based anti-cancer vaccine targeting HAAH. J Immunother Cancer 1: P210, 2013.

22. Wang K, Liu J, Yan ZL, et al: Overexpression of aspartyl(asparaginyl)-beta-hydroxylase in hepatocellular carcinoma is associated with worse surgical outcome. Hepatology 52: 164-173, 2010.

23. Tyagi S and Kramer FR: Molecular beacons: probes that fluoresce upon hybridization. Nat Biotechnol 14: 303-308, 1996.

24. Huang Z, Zhou M and Wang L: Study on the geographic distribution of liver cancer mortality and HBsAg carrier rate in China. Disease Surveillance 22: 242-245, 2007 (In Chinese).

25. Fan X, Zhao H and Zhang L: A 1:2 matched case-control study on risk factors of hepatocellular carcinoma in North Shaanxi. J Fourth Military Medical University 23: 891-895, 2002 (In Chinese).

26. Cantarini MC, De la Monte SM, Pang M, Tong M, D'Errico A, Trevisani F and Wands JR: Aspartyl-asparagyl beta hydroxylase over-expression in human hepatoma is linked to activation of insulin-like growth factor and notch signaling mechanisms. Hepatology 44: 446-457, 2006. 